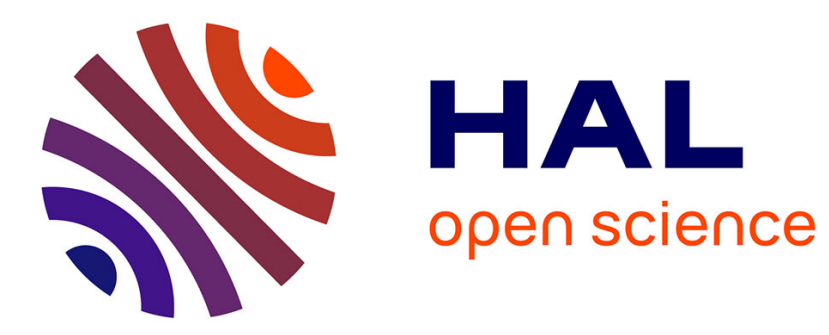

\title{
Pore-network modeling of trickle bed reactors: Pressure drop analysis
}

\author{
Rachid Hannaoui, Pierre Horgue, Faical Larachi, Yacine Haroun, Frédéric
}

Augier, Michel Quintard, Marc Prat

\section{To cite this version:}

Rachid Hannaoui, Pierre Horgue, Faical Larachi, Yacine Haroun, Frédéric Augier, et al.. Pore-network modeling of trickle bed reactors: Pressure drop analysis. Chemical Engineering Journal, 2015, vol. 262, pp. 334-343. 10.1016/j.cej.2014.09.098 . hal-01096697

\section{HAL Id: hal-01096697 https://hal.science/hal-01096697}

Submitted on 18 Dec 2014

HAL is a multi-disciplinary open access archive for the deposit and dissemination of scientific research documents, whether they are published or not. The documents may come from teaching and research institutions in France or abroad, or from public or private research centers.
L'archive ouverte pluridisciplinaire HAL, est destinée au dépôt et à la diffusion de documents scientifiques de niveau recherche, publiés ou non, émanant des établissements d'enseignement et de recherche français ou étrangers, des laboratoires publics ou privés. 


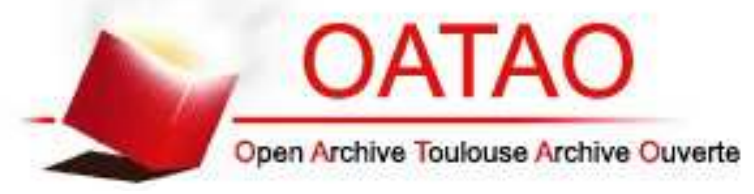

\section{Open Archive TOULOUSE Archive Ouverte (OATAO)}

OATAO is an open access repository that collects the work of Toulouse researchers and makes it freely available over the web where possible.

This is an author-deposited version published in : http://oatao.univ-toulouse.fr/ Eprints ID : 10283

To link to this article : doi: 10.1016/j.cej.2014.09.098

URL : http://dx.doi.org/10.1016/j.cej.2014.09.098

To cite this version : Hannaoui, Rachid and Horgue, Pierre and Larachi, Faical and Haroun, Yacine and Augier, Frédéric and Quintard, Michel and Prat, Marc Pore-network modeling of trickle bed reactors: Pressure drop analysis. (2015) Chemical Engineering Journal, vol. 262 . pp. 334-343. ISSN 1385-8947

Any correspondance concerning this service should be sent to the repository administrator: staff-oatao@ listes-diff.inp-toulouse.fr 


\title{
Pore-network modeling of trickle bed reactors: Pressure drop analysis
}

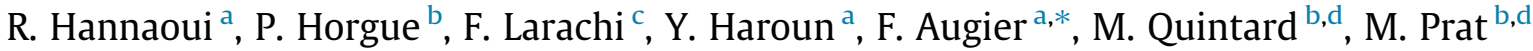 \\ a IFP Energies nouvelles-Lyon, Rond-point de l'échangeur de Solaize, BP 3, 69360 Solaize, France \\ ${ }^{\mathrm{b}}$ Université de Toulouse, INPT, UPS, Institut de Mécanique des Fluides de Toulouse, allée Camille Soula, 31400 Toulouse, France \\ ' Department of Chemical Engineering, Laval University, Québec, Québec GV 0A6, Canada \\ ${ }^{\mathrm{d}}$ CNRS, IMFT, F-31400 Toulouse, France
}

\section{H I G H L I G H T S}

- Pore network model applied to trickle bed reactors.

- Friction forces are modeled with a two-fluid Poiseuille viscous flow.

- PNM pressure drops are benchmarked using well-trodden 1D trickle bed models.

Keywords:

Pore networks

Trickle bed

Porous media

Spherical particles

Two-phase flow

Pressure drop

\begin{abstract}
A B S T R A C T
A pore network model (PNM) has been developed to simulate gas-liquid trickle flows inside fixed beds of spherical particles. The geometry has been previously built from X-ray micro-tomography experiments, and the flow in the throats between pores is modeled as a pure viscous Poiseuille two-phase flow. The flow distribution between pores and throats is obtained by solving mass and momentum balance equations. As a first application of this simple but powerful meso-scale model, a focus is proposed on the ability of PNM to estimate pressure drop and liquid saturation in co-current gas-liquid flows. PNM results are compared to the classical 1D pressure drop models of Attou et al. (1999), Holub et al. (1992) and Larachi et al. (1991). Agreement and discrepancies are discussed, and, finally, it has been found that the actual PNM approach produces realistic pressure drops as far as inertial contributions to friction are negligible. Concerning liquid saturation, the PNM only estimates its value in the throats between pores. As a consequence, liquid saturations are overestimated, but they can be easily corrected by an ad hoc empirical model.
\end{abstract}

\section{Introduction}

Catalytic fixed bed reactors are widely used in refining and chemical industry. They provide high volume fraction of catalyst while achieving near plug flow hydrodynamics. They are also easy to load and to operate. When gas and liquid reactants are involved, as in hydrotreatment or hydrodesulphurization refining processes, the operation in co-current downward flow is very useful. It may be conducted in trickling regime of liquid at the surface of catalyst particles when superficial liquid velocity is low (typically $<2 \mathrm{~cm} / \mathrm{s}$ for hydrocarbons). So-called trickle-bed reactors (TBRs) have been widely studied for decades and many reviews and books detail their global characteristics and performances [1]. Despite vessel apparent simplicity, TBR hydrodynamics is formidably complex and involves various spatial and time scales. At the particle scale

\footnotetext{
* Corresponding author
}

$(\sim \mathrm{mm})$, trickle flow depends on the shape of particles, their size, roughness, and the bed loading density. The flow also depends on the physical properties of fluids and interfaces. Local flow characteristics as the wetting efficiency of catalyst surface or the thickness of liquid film have been studied following various experimental and numerical approaches [2,3]. At the opposite extremity of the involved scales, the technology of distribution devices $(\sim \mathrm{m})$ is also an important field of research and development [4]. As perfect plug flow is sought for most applications, many studies report the effect of liquid (mal-)distribution on the global hydrodynamics and its effect on different chemical reactions [5-6]. Most of these studies report experimental investigation in cold mockups. Recently, with the steady development of appropriate computational fluid dynamics (CFD) models, numerical approaches prove to be increasingly relevant at linking the quality of fluid distribution at the top of TBRs to the flow patterns that unfold, as a result, deep inside the beds themselves [7]. Wang et al. [8] made a literature survey of trickle-bed reactors modeling 


\begin{tabular}{|c|c|c|c|}
\hline \multicolumn{4}{|c|}{ Nomenclature } \\
\hline$d$ & average particle diameter, $\mathrm{m}$ & $V_{\mathrm{sg}}$ & superficial gas velocity, $\mathrm{m} / \mathrm{s}$ \\
\hline$g$ & gravitational acceleration, $\mathrm{m} / \mathrm{s}^{2}$ & $r_{c, i j}$ & channel $i j$ constriction radius, $\mathrm{m}$ \\
\hline$l_{i j}$ & equivalent channel length, $\mathrm{m}$ & & \\
\hline$N$ & total number of pore, - & Greek & \\
\hline$P_{E}$ & network feed pressure, Pa & $\Delta t$ & time step, $\mathrm{s}$ \\
\hline$P_{F}$ & network exit pressure, $\mathrm{Pa}$ & $\Delta S_{i}$ & saturation variation, - \\
\hline$P_{i}$ & pressure of pore $i, \mathrm{~Pa}$ & $\Delta S_{\max }$ & maximum saturation variation, - \\
\hline$q_{L, i j}$ & channel ij liquid flux, $\mathrm{m}^{3} / \mathrm{s}$ & $\varepsilon_{c}$ & average liquid saturation calculated by PNM, - \\
\hline$q_{G, i j}$ & channel ij gas flux, $\mathrm{m}^{3} / \mathrm{s}$ & $\varepsilon_{L}$ & average liquid saturation after correction, - \\
\hline$q_{G i(F)}$ & $\begin{array}{l}\text { gas flux coming from feed manifold to entrance bound- } \\
\text { ary pore } i, \mathrm{~m}^{3} / \mathrm{s}\end{array}$ & & $\begin{array}{l}\text { liquid viscosity, Pa s } \\
\text { gas viscosity, Pa s }\end{array}$ \\
\hline$q_{L i(F)}$ & $\begin{array}{l}\text { liquid flux coming from feed manifold to entrance } \\
\text { boundary pore } i, \mathrm{~m}^{3} / \mathrm{s}\end{array}$ & $\Gamma_{z-}$ & $\begin{array}{l}\text { lower network domain exit boundary } \\
\text { upper network domain entrance boundary }\end{array}$ \\
\hline$q_{G i(E)}$ & $\begin{array}{l}\text { gas flux coming from exit boundary pore } i \text { to exit man- } \\
\text { ifold, } \mathrm{m}^{3} / \mathrm{s}\end{array}$ & $\begin{array}{l}\rho_{L+} \\
\rho_{G}\end{array}$ & $\begin{array}{l}\text { liquid density, } \mathrm{kg} / \mathrm{m}^{3} \\
\text { gas density, } \mathrm{kg} / \mathrm{m}^{3}\end{array}$ \\
\hline$q_{L i(E)}$ & $\begin{array}{l}\text { liquid flux coming from exit boundary pore } i \text { to exit } \\
\text { manifold } \mathrm{m}^{3} / \mathrm{s}\end{array}$ & & \\
\hline$Q_{L 0}$ & cumulative (feed) liquid flow rate, $\mathrm{m}^{3} / \mathrm{s}$ & Subscript & \\
\hline$Q_{G 0}$ & cumulative (feed) gas flow rate, $\mathrm{m}^{3} / \mathrm{s}$ & $\begin{array}{l}l, j \\
\mathrm{c}\end{array}$ & $\begin{array}{l}\text { pore indices } \\
\text { channel }\end{array}$ \\
\hline$S_{i}$ & pore $i$ liquid saturation, - & E & exit \\
\hline & channel $i j$ liquid saturation, - & $\mathrm{F}$ & feed \\
\hline $\begin{array}{l}S_{\text {network }} \\
V_{i}\end{array}$ & $\begin{array}{l}\text { average saturation, - } \\
\text { pore } i \text { volume, } \mathrm{m}^{3}\end{array}$ & $\mathrm{p}$ & pore \\
\hline$V_{\mathrm{sl}}$ & superficial liquid velocity, $\mathrm{m} / \mathrm{s}$ & & \\
\hline
\end{tabular}

and pointed out two main CFD methods based on an Eulerian description, where gas and liquid are treated as interpenetrating continua: volume of fluid method and Euler-Euler method. In the first, a surface tracking technique is used to solve for the gas-liquid interface. This method requires however a high refined mesh resolution, which makes the method applicable only for relatively small-scale beds $[3,9,10]$. The second, based on an averaging method of local gas and liquid mass and momentum conservation equations, leads to an "effective porous medium" representation [8]. Since these models do not simulate directly the flow over the actual physical geometry, this CFD approach needs closure laws concerning interaction between fluids $(\mathrm{G} / \mathrm{L}, \mathrm{L} / \mathrm{S}, \mathrm{G} / \mathrm{S})$, turbulence (if any) and dispersion mechanisms. It must be emphasized that the development of appropriate physical models is still a huge challenge.

Another field of research associated to global hydrodynamics inside TBRs concerns the prediction of macroscopic properties as global pressure drop and fluid volume fraction (or saturation). Studies are based on mock-up experiments assuming generally a perfect plug flow. These models are of first importance for catalyst and process licensors, because they guide the choice of catalyst particle sizes, shapes and method of loading, that have to be accurately calibrated for each application. Several suggestions of 1D pressure drop models are available in literature. Ergun-like models as well as empirical models are widely used [11-14]. Based on experimental investigation, Larachi et al. [11] proposed an empirical correlation for pressure drop and liquid hold-up in trickle bed reactors. Ergun-like models are mostly inspired from the phenomenological single-phase Ergun empirical representation assuming different hydraulic diameters for gas and liquid when these latter are in contact. On that basis, Holub et al. [15] suggested viscous and inertial resistances that depend on liquid saturation, bed geometrical characteristics (void and specific surface area) as well as Ergun constants. However, the resulting macroscopic model does not take into account gas-liquid interactions that especially build up when gas and/or liquid inertia increase. Attou et al. [13] built a phenomenological macroscopic hydrodynamic model in trickle-bed reactors based on the balance of forces exerted on both phases at particle scale. Within this formalism, the suggested porous resistance that applies to liquid is weighted by the medium tortuosity that was itself estimated as the reciprocal of liquid saturation. In the present work, the pressure drop model of Attou et al. [13] is chosen as a reference because of its comprehensiveness in accounting for the porous resistances as well as gas-liquid interactions. However, model predictions have always to be considered carefully, especially when models are forced to venture outside from their range of validation. For this reason, comparison to other models, such as Holub et al. [15] and Larachi et al. [16] is also proposed.

At spatial scales intermediate between particle and vessel scales, hydrodynamics is often poorly described in the literature. This lack of knowledge is due to the difficulty to investigate experimentally these "meso"-scales of several hundred to several thousand of particles. Powerful experimental tools consisting of non-intrusive techniques as magnetic resonance imaging (MRI) or (non-)ionizing tomographies are being currently used with relative success to describe the flow at different scales [17-19]. As an alternative to such sophisticated experiments, CFD models based on reconstruction of fluid interfaces may be an efficient approach in the future to investigate meso-scales though at present they are limited to cells of a few number of particles $[3,20]$.

An intermediate approach, based on the method of pore network modeling (PNM), is developed in this study. Following this approach, the structure of the fixed bed is modeled as a network of discrete pores linked by different channels or "throats" of different sizes [21-27]. As an intermediate semi-empirical approach, the flow is modeled in the throats following a simple physical model detailed in the next section whereas the distribution of fluids between pores and throats is simulated by solving mass and pressure balances at the scale of the calculated domain. Benefits of this approach are simplicity and low CPU time on top of delivering relatively high quality simulations of fixed beds consisting of thousands of particles [28]. On the other hand, an accurate description of the geometry is required, as well as the validation of the 
local hydrodynamic model inside channels. In our previous work, the PNM approach has been successfully applied to single-phase flows inside fixed bed reactors [28]. The PNM geometry used in the previous study has been extracted from X-ray micro-tomography measurements and the resulting good ability for predicting single phase pressure drop was a prerequisite to the modeling of more complex two-phase flows.

In this context, the objectives of the presented work are to apply the PNM approach to gas-liquid trickle flows in fixed-bed reactors and to evaluate the consistency of resulting flow predictions. To do that, a numerical model based on the calculation of two-fluid Poiseuille profiles has been implemented in the PNM. The twofluid resulting PNM has been then used to calculate pressure drop and liquid saturation in a domain with various properties of the fixed bed and fluids. A comparison to classic 1D pressure drop methods is presented and discussed.

\section{Models}

Network models are widely used to study the nature of fluid flow from the pore $(\mu \mathrm{m})$ to core $(\mathrm{mm}$ to $\mathrm{cm})$ scale. It was used for several applications such as chemical engineering, petroleum engineering, physics and hydrology. Pore network models involving two phase flows can be divided into quasi-static and dynamic ones. The majority belongs to the first category and are generally based on the capillary equilibrium assumption. They can simulate only successive stationary states ignoring dynamic aspects of pressure propagation and interface dynamics [29-32]. However, the second (used in this work) can simulate transient behavior of flow with time. It is used extensively as an up-scaling tool, as it is relatively simple and computationally less demanding than the other methods, i.e., for example direct numerical simulations at the pore-scale. Several dynamic pore-network models have been developed for various applications [33-38] and The pore space can be discretized following two main approaches: the first one focuses on the throats where all volume is assumed to be distributed among the throats [39] and pores are just intersections between them; the second approach (used in this work), assumes that the volume is distributed among the pores while throats are treated as inter-poral volumeless connections [40]. The majority of dynamic pore networks have a regular structure with fixed coordination number making them computationally simpler than the irregular unstructured networks. In this work, we have developed a realistic dynamic pore network model where the network was extracted from a real porous medium by micro-tomography technique [28].

\subsection{Network geometry}

Generally, pore network modeling uses the network of pores interconnected by narrow spaces referred to as pore throats to model the void space of a porous medium. The majority of dynamic pore networks have a regular structure because it is easy to implement. But, in real porous media, the grains are packed in a disordered manner rather than occupying a regular lattices, hence our efforts to incorporate these features in our model.

A sample porous medium was prepared by packing 490 monodisperse 4-mm spherical glass beads in a small container with an overall bed porosity of $36 \%$. The size of the reconstructed domain was $3.06_{x} \times 2.76_{y} \times 3.04_{z} \mathrm{~cm}^{3}$ with a unitary resolution $\Delta x \times$ $\Delta y \times \Delta z=40^{3} \mu \mathrm{m}^{3}$ and a 3D image composed of $765 \times 690 \times$ 760 voxels. A pore network extraction methodology developed by Youssef and coworkers $[41,42]$ was subsequently applied to convert the 3D density maps into resolved pore space. The method enables the partitioning of the pore space into pore bodies and throats, according to the following 4-step procedure: segmentation, skeletonization, pore space partitioning (and throat detection) and parameters extraction. As detailed in [28], pore bodies recover the total volume between solid particles. Pores are connected by throats, and throat radii are defined as those describing the largest collapsible sphere to be inscribed in the throat cross section. The throats are considered null volume, except for the pressure drop calculation, where distances between pore centroids are taken into account.

The geometry is described in detail in [28]. It was successfully used to study inertial effects in single-phase flows. In this work, we are interested in fixed-bed reactors with $2 \mathrm{~mm}$ glass beads. For this purpose, we used a homothetic transformation by multiplying the network geometrical lengths and measures by a scaling constant $H$ defined as the ratio between target and initial grain diameters.

Fig. 1a represents a binary 3D image of each phase (solid and void) in our porous medium. Fig. 1b and c are 3D illustrations of the pore network extracted using the methodology described in [28]. The specificities of the bead pack are clearly represented in terms of pore connectivities, throat lengths and radii, and pore bodies as depicted by their isovolume spherical proxies in Fig. 1c. One can note that the porous medium exhibits a very highly irregular geometry.

\subsection{Two phase flow in throat}

It is assumed that two incompressible and immiscible fluids are displacing each other in the network under a pressure gradient
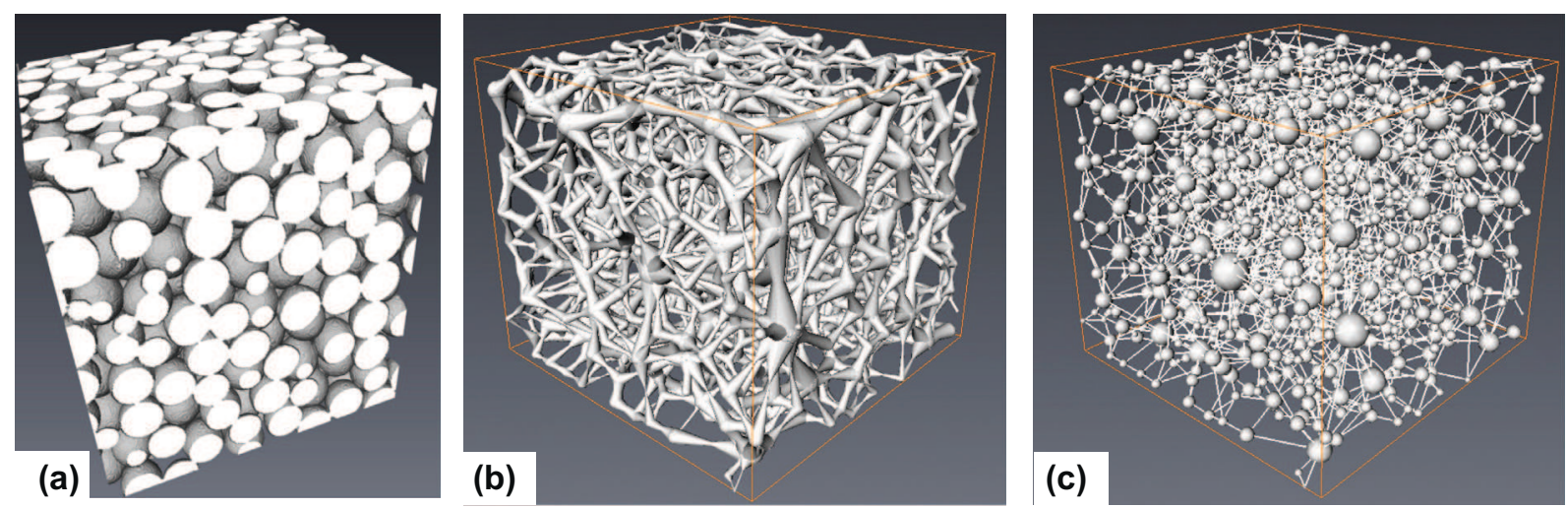

Fig. 1. Pore network modelling: (a) Reconstruction of the porous parallelepipedic specimen sampled using 2D micro-tomography scans. (b) Representation of the pore network in terms of pore connectivities, throat radii and lengths. (c) Pore volumes of equivalent spheres (from Larachi et al. (2014) [28]). 
driving force and gravity contribution. The volume contributed by the pore throat is assumed to be negligible because relatively small compared to the volumes of pore bodies. Therefore, as explained in Section 2, the model attributes all the volume to the pore bodies and the motion of an interface through a pore throat is assumed to occur instantaneously. Furthermore, the local capillary pressure in the pore bodies is assumed to be negligible, so that the two phase pressures are equal whatever the local saturation of that pore body, as it is assumed in the model of Attou et al. [13] for instance. This assumption is motivated by the relatively high value of the Bond number (Bo $>1$ ), and the admittedly "weak interaction" trickling regime. A low Reynolds number flow is considered and inertial effects can be neglected. Laminar viscous dissipation within the pore-throats are accounted for by assuming a two-phase Poiseuille flow globally portraying the well-known generalized Darcy model in the overall bed pressure gradient. The volumetric flux is related to pressures at the pore bodies by the following two-phase Poiseuille's law (as sketched in Fig. 2), for gas:

$$
\begin{aligned}
q_{G, i j}= & -\frac{\pi r_{c, i j}^{4}}{8 \mu_{L} l_{i j}}\left[\frac{\mu_{L}}{\mu_{G}}\left(1-s_{i j}\right)^{2}+2 s_{i j}\left(1-s_{i j}\right)\right]\left[\left(P_{i}-P_{j}\right)+g \rho_{G}\left(z_{i}-z_{j}\right)\right] \\
& -\frac{\pi r_{c, i j}^{4}}{8 \mu_{L} l_{i j}} g\left(z_{i}-z_{j}\right)\left(\rho_{L}-\rho_{G}\right)\left[2 s_{i j}\left(1-s_{i j}\right)+2\left(1-s_{i j}\right)^{2} \ln \left(1-s_{i j}\right)\right],
\end{aligned}
$$

and for liquid

$$
\begin{aligned}
q_{L, i j}= & -\frac{\pi r_{c, i j}^{4} s_{i j}^{2}}{8 \mu_{L} l_{i j}}\left[\left(P_{i}-P_{j}\right)+g \rho_{L}\left(z_{i}-z_{j}\right)\right] \\
& +\frac{\pi r_{c, i j}^{4}}{8 \mu_{L} l_{i j}} g\left(\rho_{L}-\rho_{G}\right)\left(z_{i}-z_{j}\right)\left[2 s_{i j}\left(1-s_{i j}\right)+2\left(1-s_{i j}\right)^{2} \ln \left(1-s_{i j}\right)\right],
\end{aligned}
$$

where $\mu_{\alpha}$ and $\rho_{\alpha}$ are the dynamic viscosity and density of $\alpha$ fluid moving in the throat, respectively, $P_{i}$ and $P_{j}$ are the static pressure in adjacent pores $i$ and $j$ at vertical positions $z_{i}$ and $z_{j}$, respectively, $s_{i j}$ is the liquid local pore throat saturation (related to the film thickness) and $g$ is the gravity constant. $r_{c, i j}$ are a cylindrical pore throat radius and $L_{i j}$ are the length between the two pore centers.

The saturation in a pore-throat is related to the saturation of the neighboring pores and can be computed by several approaches. In the present study, the focus is put on the pressure drop and to ensure stability to the simulations, it is assumed that the saturation $s_{i j}$ in a pore throat is equal to the saturation of the upstream pore body.

Volumetric fluxes are treated as algebraic quantities to handle the various flow modalities, i.e., the (positive) fluxes leading into pore $i$ from an upstream pore $j$ are discriminated from those (negative) leading out of pore $i$ into a downstream pore $j$. Regardless of local direction of flow, the per-phase total head losses are

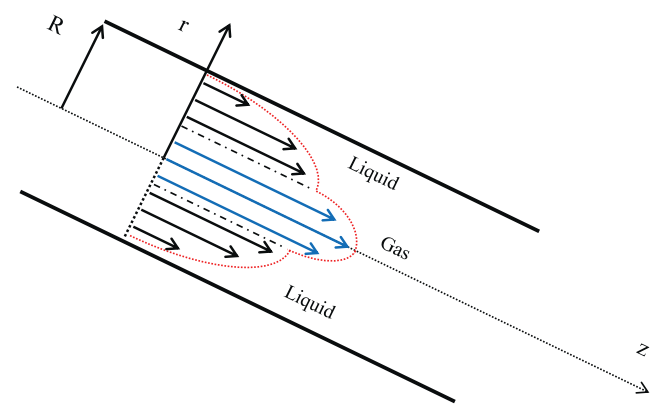

Fig. 2. Scheme of a two-fluid axisymmetric concurrent Poiseuille profile of velocity in a channel. computed as the per-phase total head from upper pore $i$ minus that for lower pore $j$. Such directional behavior of fluxes also accounts for the fact that not all throats in the network will discharge in a descending manner, despite prevalence of a macroscopic downflow. Hence, whether the least penalizing flow across throat is upwards or downwards, the model handles local descending and ascending instances alike.

\subsection{Pore-level mass balance}

Considering a gas-liquid two-phase flow, the two mass conservation equations can, be reformulated as a total volumetric balance equation for the pore body $i$, given by:

$$
\sum_{j=1}^{n} q_{L, i j}+\sum_{j=1}^{n} q_{G, i j}=0, \quad i=1,2, \ldots, N,
$$

With $n$ the number of connected pores throats and $N$ the number of pores. The liquid mass balance reads:

$V_{i} \frac{\partial S_{i}}{\partial t}=\sum_{j=1}^{n} q_{L, i j}, \quad i=1,2, \ldots, N$,

where $S_{i}$ and $V_{i}$ are the liquid saturation and volume of pore body respectively. The numerical resolution of this two equations system is described in Section 2.5.

\subsection{Network boundary conditions}

Fig. 3 shows the boundary conditions adopted to handle the top feed and bottom exit fluid streams traversing the pore network. To mimic as closely as possible actual fluid feed/discharge from trickle beds, the exit pressure $P_{E}$ is assumed to be known a priori, whereas the cumulative fluid flow rates, $Q_{L O}$ and $Q_{G 0}$, are imposed at the entrance in a co-current down-flow setting along the $z$-direction.

The pore bodies occupying $\Gamma_{z^{+}}$boundary (called $\Gamma_{z^{+}}$-pores) are powered from the feed manifold (viewed as an entrance megapore) positioned atop at elevation $z_{F}$, where from the total payload is delivered at a constant feed pressure $P_{F}$ and saturation $S_{F}$. The manifold pressure and saturation are obtained by solving the mass conservation equations with additional injection source terms:

$Q_{L 0}+\sum_{j \in \Gamma_{z+}} q_{L j(F)}=0$

$Q_{L 0}+Q_{G 0}+\sum_{j \in \Gamma_{z+}} q_{L j(F)}+\sum_{j \in \Gamma_{z+}} q_{G j(F)}=0$

$Q_{L 0}+\sum_{j \in \Gamma_{z+}} q_{L j(F)}=0$,

Note that liquid mass conservation (Eq. (6)) does not contain accumulation term (contrary to Eq. (4)) since the saturation in the manifold is only computed to satisfy the conservation between injection and $\Gamma_{z^{+}}$-pores flow rates.

Likewise, the pore bodies occupying the $\Gamma_{z-}$ boundary deliver their fractional flow rates to an exit receptacle (viewed as an exit mega-pore) located at elevation $z_{E}$ kept at a known exit pressure $P_{E}$ (Fig. 3). The boundary conditions of the four remaining vertical sides were no-flow boundaries. Mass conservation around feed manifold is given as,

$$
\begin{aligned}
q_{L i(F)}= & -\frac{\pi r_{\Gamma_{Z+}, i}^{4} s_{F}^{2}}{8 \mu_{L}\left(z_{F}-z_{\Gamma_{Z+}, i}\right)}\left[\left(P_{F}-P_{\Gamma_{Z+}, i}\right)+g \rho_{L}\left(z_{F}-z_{\Gamma_{Z+}, i}\right)\right] \\
& +\frac{\pi r_{\Gamma_{Z+}, i}^{4}}{8 \mu_{L}} g\left(\rho_{L}-\rho_{G}\right)\left[2 s_{F}\left(1-s_{F}\right)+2\left(1-s_{F}\right)^{2} \ln \left(1-s_{F}\right)\right]
\end{aligned}
$$

and exit receptacle as: 


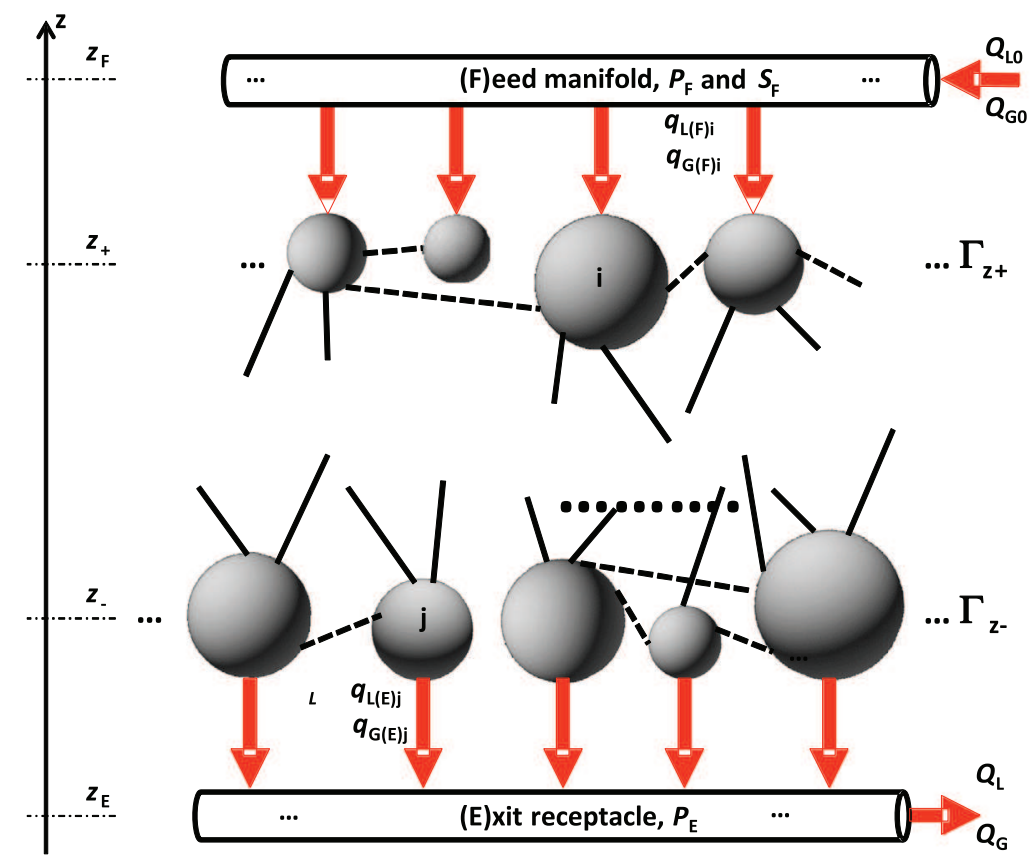

Fig. 3. Illustration of the PNM boundary conditions.

$$
\begin{aligned}
& q_{G i(E)}=-\frac{\pi r_{\Gamma_{--}, i}^{4}}{8 \mu_{L}\left(z_{\Gamma-, i}-z_{E}\right)}\left[\frac{\mu_{L}}{\mu_{G}}\left(1-s_{\Gamma_{Z_{-}}, i}\right)^{2}+2 s_{\Gamma_{Z_{-}, i}}\left(1-s_{\Gamma_{Z_{-}}, i}\right)\right] \\
& \times\left[\left(P_{\Gamma_{Z_{-}}, i}-P_{E}\right)+g \rho_{G}\left(z_{\Gamma-, i}-z_{E}\right)\right]-\frac{\pi r_{\Gamma_{Z-}, i}^{4}}{8 \mu_{L}} g\left(\rho_{L}-\rho_{G}\right) \\
& \times\left[2 s_{\Gamma_{Z_{-}, i}}\left(1-s_{\Gamma_{Z_{-}}, i}\right)+2\left(1-s_{\Gamma_{Z_{-}}, i}\right)^{2} \ln \left(1-s_{\Gamma_{Z_{-}}, i}\right)\right] \\
& q_{L i(E)}=-\frac{\pi r_{\Gamma_{--}, j}^{4} s_{\Gamma_{Z-}, i}^{2}}{8 \mu_{L}\left(z_{\Gamma-, i}-z_{E}\right)}\left[\left(P_{\Gamma_{Z_{-}, i}}-P_{E}\right)+g \rho_{G}\left(z_{E}-z_{\Gamma_{Z_{-}}, j}\right)\right] \\
& +\frac{\pi r_{\Gamma_{-}, i}^{4}}{8 \mu_{L}} g\left(\rho_{L}-\rho_{G}\right)\left[2 s_{\Gamma_{Z_{-}, j}}\left(1-s_{\Gamma_{Z_{-}, i}}\right)\right. \\
& +2\left(1-s_{\Gamma_{Z_{-}}, i}\right)^{2} \ln \left(1-s_{\Gamma_{z_{-}}, i}\right),
\end{aligned}
$$

Finally, flux conservation around pore $i$ belonging to boundary $\Gamma_{z^{+}}$(respectively, $\Gamma_{z-}$ ) interconnected to $n$ pores can be written as:

$$
\begin{aligned}
& q_{L i(F)}+q_{G i(F)}+\sum_{j=1}^{n} q_{L, i j}+\sum_{j=1}^{n} q_{G, i j}=0 \\
& q_{L i(E)}+q_{G i(E)}+\sum_{j=1}^{n} q_{L, i j}+\sum_{j=1}^{n} q_{G, i j}=0
\end{aligned}
$$

Note that the gravity term is written to account for the whole fluid mass in the pore and not only that in the channel, thus the pore centroid difference $z_{i}-z_{j}$. The frictional overall pressure gradient across the whole pore network body is obtained as the difference between the volume-average hydraulic heads at $\Gamma_{z^{+}}$and $\Gamma_{z-}$ boundaries [37] divided by their corresponding average separating distance.

\subsection{Numerical resolution}

The total mass conservation equations (Eqs. (3) and (5)) coupled with flow rate expressions (Eqs. (1), (2) and (5)-(10)) leads to a system of $N+1$ equations and $N+1$ unknowns for the pore pressures, where $N$ is the number of pores. Assuming the pore pressures are uniform, the system can be solved to determine the pressure at each time step. This is accomplished by solving the matrix equation,

$A \vec{P}=\vec{B}$,

where $A$ is the conductance matrix, $\vec{P}$ is the pressure vector, and $\vec{B}$ contains the pressure at the outlet boundary, the gravity terms and the injection source term (for the "manifold" pore). Nonlinearities due to the two-phase Poiseuille laws are treated explicitly by using the last known saturation field [43]. The linear system of equations (Eq. (12)) is solved by Generalized Minimum Residual method using the SPARSKIT mathematical library [44].

Then, the system constituted by liquid mass conservation equations (Eqs. (4)) is explicitly solved using the previously computed pressure field to get the new saturation field:

$V_{i} \frac{s_{i}^{t+1}-s_{i}^{t}}{\Delta t}=\sum_{j=1}^{n} q_{L, i j}^{t}$

where $S_{i}$ and $V_{i}$ are the liquid saturation and volume of pore body, respectively. This equation is explicitly discretized to calculate the new saturation using pressure fields and old saturation. Then the saturation at the iteration $t+1$ is given by: $\mathrm{f}$

$S_{i}^{t+1}=S_{i}^{t}+\frac{\Delta t}{V_{i}}\left(\left(A_{L}^{t} P^{t}\right)_{i}-B_{L, i}^{t}\right)$

The saturation of gas phase for pore $i$ is given by $1-S_{i}$.

Finally, the saturation in the manifold $S_{F}$ ensuring liquid conservation between the inlet and the outlets of the entrance pore is calculated by iterative method. As a consequence, once the global PNM converged, the saturation in the pores is equal to the saturation in downstream throats.

\subsection{Time step}

As saturation is explicitly computed, it is necessary to limit the time-step to ensure stability to the simulations. The time-step limitation consists in fixing a maximal variation of saturation $\Delta S_{\max }$ that can occurs during one iteration. The time-step is the minimum 
filling time found among pores and throats containing a moving interface [45]:

$\Delta t=\min \left(\frac{V_{i} \Delta S_{\max }}{\left|\sum_{j=1}^{n} q_{L, i j}\right|}\right)$.

However, to determine this time step, this approach requires the computation of the sum of the fluxes for all network element every time and increases substantially CPU time. We therefore opted for an adaptive time step strategy with the following algorithm:

1. Impose an initial time step and a permissible maximum variation of saturation $\Delta S_{\max }$ that leads to stabilize the calculation.

2. Evaluate the pressures and saturations.

3. If $\max \left(\Delta S_{i}\right) \leqslant \Delta S_{\max }$ continue to the next time step.

4. If $\max _{i}^{i}\left(\Delta S_{i}\right)>\Delta S_{\max }$, then $\Delta t^{\text {new }}=\frac{\Delta t^{\text {old }}}{2}$, go to 2 .

5. If the time step is not changed after 10 iterations, then $\Delta t^{\text {new }}=2 \Delta t^{\text {old }}$

6. No saturation changes in pores $\left(\Delta S_{\max }<10^{-6}\right)$ means that the steady state is reached.

This algorithm prevents calculation of Eq. (15) every time step and for all the network elements.

A typical computation time is approximately $30 \mathrm{~min}$ on a dual core 3 Go RAM Intel Xeon $2.8 \mathrm{~Hz}$. For the sake of comparison, a two-fluid (VOF) CFD simulation on the same geometry would require several days of simulations on a parallel 128 cores calculator.

\subsection{Averaging procedure}

Our simulation result in local-scale variables such as static pressures, saturations, and fluxes. To obtain the macroscopic variables, we have to average these local variables over the network. Average saturation is defined as follows [46]:

$S_{\text {network }}=\frac{v^{L}}{v^{G}+v^{L}}=\frac{\sum_{i=1}^{N} S_{i} V_{i}}{\sum_{i=1}^{N} V_{i}}$

where $v^{L}$ and $v^{G}$ are the total volume of liquid and gas, respectively. The macroscopic head loss gradient is given by [43]:

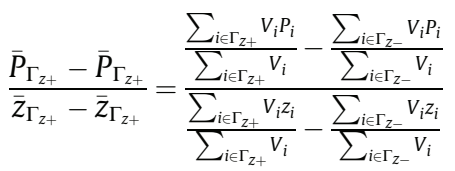

\section{Results and discussion}

The PNM has been used in various conditions in order to evaluate its ability to predict pressure drops in two-phase trickling flow conditions. As a preliminary task, the throat effective aspect ratio has to be stated once and for all, in order to deliver a realistic pressure drop. This point is detailed in the next paragraph. Then the initial geometry, determined by X-ray micro-tomography from a loading of $4 \mathrm{~mm}$ spherical glass particles [28] was homothetically modified in order to simulate loading of particles of different sizes ranging from 0.5 to $2 \mathrm{~mm}$. For each geometry, the same throat effective aspect ratio is used. Various liquid and gas physical properties are used in a wide range of superficial gas and liquid velocities. Table 1 summarizes all the simulations performed using the PNM along with Holub et al., Attou et al. and Larachi et al. estimation methods. The effects of separate operating parameters on the pressure drop are discussed one by one in the following sections.
The reference case is $d=1 \mathrm{~mm}, V_{\mathrm{sl}}=0.002 \mathrm{~m} / \mathrm{s}, V_{\mathrm{sg}}=0.02 \mathrm{~m} / \mathrm{s}$, $\mu_{L}=1.10^{-3}$ Pa s, $\mu_{G}=2.10^{-5}$ Pa s, $\rho_{G}=15 \mathrm{~kg} / \mathrm{m}^{3}, \quad \rho_{L}=750 \mathrm{~kg} / \mathrm{m}^{3}$ and one-at-a-time parameter change strategy is adopted to probe model responses nearby the given reference case.

\subsection{Throat effective aspect ratio}

In this work, and as previously described in Section 2.2, the geometries of pore throat cross-sections have been idealized as cylinders of circular cross-sections. As witnessed from Fig. 2 poral morphology, such idealization is indeed far from realistic and induces errors in some applications of predictive pore network models as relative permeability $[29,30,47]$ and macroscopic head loss gradient [28]. It has been shown that the throat cylindrical model with a circular equivalent radius ignores the Venturi-like pore-throat geometry. In our previous work [28], we introduced a correction, namely a throat effective aspect ratio, $r_{\text {ecij }} / r_{c i j}$, to take into account such deviations from the cylindrical shape. There exists several manners to obtain this correction [28]. In this work, we have chosen to assign a single $r_{e c i j} / r_{c i j}$ value to all pore throats and to estimate its value by comparison of the PNM result to an experimental determination of the bed pressure loss for given volumetric flow rates in the Darcy flow regime. The aspect ratio would correspond to that value which minimizes the error between measured and PNM simulated pressure loss. The results are illustrated in Fig. 4 for a $1 \mathrm{~mm}$ glass beads mono-disperse packing, liquid superficial velocity $\left(V_{\mathrm{sl}}=0.002 \mathrm{~m} / \mathrm{s}\right)$ and gas superficial velocity $\left(V_{\mathrm{sg}}=0.02 \mathrm{~m} / \mathrm{s}\right)$, the calculations conditions are given in Table 1 . This yields a throat effective aspect ratio of 0.83 that minimizes the error between the Attou et al. model [13] and the pore network model prediction.

Fig. 5 illustrates a PNM simulation of liquid saturation (on the left) and pressure (on the right) fields in the reference conditions. Slight heterogeneities of liquid local saturation illustrate the ability of the PNM approach to deviate from the initial homogenous boundary conditions. Recall that PNM simulated pore saturation field, once steady-state solutions are reached, corresponds conceptually to the field of downstream throat saturations.

\subsection{Effect of liquid superficial velocity}

The liquid superficial velocity is changed from the reference case, from 0.002 to $0.007 \mathrm{~m} / \mathrm{s}$. A comparison with Attou et al., Holub et al. and Larachi et al. estimation methods is displayed in Fig. 6. PNM results are rather in good agreement with the Attou et al. model over the entire $V_{\mathrm{sl}}$ range. The best fit observed at low velocity is expected in accordance with the throat calibration of the effective aspect ratio in the Darcy range. However, where the maximal deviation from the Attou et al. model does not exceed $10 \%$, the other remaining pressure drop methods differ from $\pm 50 \%$, while they nonetheless present similar qualitative evolutions as a function of liquid velocity. The exercise herewith described points to the premature character of the PNM to fully predict two-phase flow pressure field in a trickle bed without an acceptable $a$ priori estimate of the pore throat aspect ratio. Assuming unit circularity would have led, according to Fig. 4, to an estimated error of 30$40 \%$ on pressure drops which is in the range of the errors by Holub et al. and Larachi et al. methods.

\subsection{Effect of gas superficial velocity}

The gas superficial velocity is changed in the range from 0.02 to $0.2 \mathrm{~m} / \mathrm{s}$ and compared in Fig. 7 with the existing chosen estimation tools. The Attou et al. model reflects in a much important impact of $V_{\text {sg }}$ than Holub et al. or Larachi et al. estimation methods. Here again, the PNM pressure drop value at low gas velocity is in close 
Table 1

Results of PNM, Attou et al., Holub et al. and Larachi et al. estimation methods in various conditions.

\begin{tabular}{|c|c|c|c|c|c|c|c|c|c|c|c|c|c|c|}
\hline \multicolumn{7}{|c|}{ Conditions } & \multicolumn{4}{|l|}{$\mathrm{DP}(\mathrm{Pa} / \mathrm{m})$} & \multicolumn{4}{|c|}{ Liquid saturation } \\
\hline $\begin{array}{l}d \\
(\mathrm{~mm})\end{array}$ & $\begin{array}{l}\rho_{G}(\mathrm{~kg} / \\
\mathrm{m} 3)\end{array}$ & $\begin{array}{l}\rho_{L}(\mathrm{~kg} / \\
\mathrm{m} 3)\end{array}$ & $\begin{array}{l}\mu_{G}(\mathrm{~kg} / \\
\mathrm{m} 3)\end{array}$ & $\begin{array}{l}\mu_{\mathrm{L}}(\mathrm{kg} / \\
\mathrm{m} 3)\end{array}$ & $\begin{array}{l}V_{\mathrm{sg}}(\mathrm{m} / \\
\mathrm{s})\end{array}$ & $\begin{array}{l}V_{\mathrm{sl}}(\mathrm{m} / \\
\mathrm{s})\end{array}$ & PNM & Attou & Holub & Larachi & PNM & Attou & Holub & Larachi \\
\hline 1 & 15 & 750 & 0.00002 & 0.001 & 0.02 & 0.002 & $1.99 \mathrm{E}+04$ & $2.17 \mathrm{E}+04$ & $1.21 \mathrm{E}+04$ & $3.34 \mathrm{E}+04$ & 0.70 & 0.54 & 0.56 & 0.47 \\
\hline 1 & 15 & 750 & 0.00002 & 0.001 & 0.02 & 0.003 & $2.64 \mathrm{E}+04$ & $2.69 E+04$ & $1.55 E+04$ & $4.01 \mathrm{E}+04$ & 0.74 & 0.57 & 0.61 & 0.50 \\
\hline 1 & 15 & 750 & 0.00002 & 0.001 & 0.02 & 0.004 & $3.28 \mathrm{E}+04$ & $3.17 \mathrm{E}+04$ & $1.88 \mathrm{E}+04$ & $4.56 \mathrm{E}+04$ & 0.76 & 0.60 & 0.64 & 0.53 \\
\hline 1 & 15 & 750 & 0.00002 & 0.001 & 0.02 & 0.005 & $3.88 \mathrm{E}+04$ & $3.62 \mathrm{E}+04$ & $2.19 \mathrm{E}+04$ & $5.04 \mathrm{E}+04$ & 0.78 & 0.62 & 0.66 & 0.55 \\
\hline 1 & 15 & 750 & 0.00002 & 0.001 & 0.02 & 0.006 & $4.48 \mathrm{E}+04$ & $4.06 \mathrm{E}+04$ & $2.51 \mathrm{E}+04$ & $5.48 \mathrm{E}+04$ & 0.80 & 0.64 & 0.68 & 0.57 \\
\hline 1 & 15 & 750 & 0.00002 & 0.001 & 0.02 & 0.007 & $5.08 \mathrm{E}+04$ & $4.49 E+04$ & $2.82 \mathrm{E}+04$ & $5.87 \mathrm{E}+04$ & 0.81 & 0.65 & 0.69 & 0.58 \\
\hline 1 & 15 & 750 & 0.00002 & 0.001 & 0.02 & 0.002 & $1.99 \mathrm{E}+04$ & $2.17 E+04$ & $1.21 \mathrm{E}+04$ & $3.34 \mathrm{E}+04$ & 0.70 & 0.54 & 0.56 & 0.47 \\
\hline 1 & 15 & 750 & 0.00002 & 0.001 & 0.04 & 0.002 & $2.60 \mathrm{E}+04$ & $3.24 \mathrm{E}+04$ & $1.72 \mathrm{E}+04$ & $3.93 \mathrm{E}+04$ & 0.62 & 0.48 & 0.51 & 0.44 \\
\hline 1 & 15 & 750 & 0.00002 & 0.001 & 0.06 & 0.002 & $3.10 \mathrm{E}+04$ & $4.33 E+04$ & $2.25 E+04$ & $4.38 \mathrm{E}+04$ & 0.57 & 0.44 & 0.47 & 0.42 \\
\hline 1 & 15 & 750 & 0.00002 & 0.001 & 0.08 & 0.002 & $3.56 \mathrm{E}+04$ & $5.46 \mathrm{E}+04$ & $2.81 \mathrm{E}+04$ & $4.76 \mathrm{E}+04$ & 0.53 & 0.41 & 0.44 & 0.40 \\
\hline 1 & 15 & 750 & 0.00002 & 0.001 & 0.1 & 0.002 & $3.99 \mathrm{E}+04$ & $6.67 \mathrm{E}+04$ & $3.39 \mathrm{E}+04$ & $5.09 \mathrm{E}+04$ & 0.50 & 0.39 & 0.41 & 0.39 \\
\hline 1 & 15 & 750 & 0.00002 & 0.001 & 0.12 & 0.002 & $4.39 E+04$ & $7.94 \mathrm{E}+04$ & $4.02 \mathrm{E}+04$ & $5.39 \mathrm{E}+04$ & 0.48 & 0.37 & 0.39 & 0.38 \\
\hline 1 & 15 & 750 & 0.00002 & 0.001 & 0.14 & 0.002 & $4.77 \mathrm{E}+04$ & $9.28 \mathrm{E}+04$ & $4.67 \mathrm{E}+04$ & $5.67 \mathrm{E}+04$ & 0.46 & 0.35 & 0.38 & 0.38 \\
\hline 1 & 15 & 750 & 0.00002 & 0.001 & 0.16 & 0.002 & $5.15 \mathrm{E}+04$ & $1.07 \mathrm{E}+05$ & $5.37 \mathrm{E}+04$ & $5.93 \mathrm{E}+04$ & 0.44 & 0.34 & 0.36 & 0.37 \\
\hline 1 & 15 & 750 & 0.00002 & 0.001 & 0.18 & 0.002 & $5.50 \mathrm{E}+04$ & $1.22 \mathrm{E}+05$ & $6.10 \mathrm{E}+04$ & $6.17 \mathrm{E}+04$ & 0.43 & 0.32 & 0.35 & 0.37 \\
\hline 1 & 15 & 750 & 0.00002 & 0.001 & 0.2 & 0.002 & $5.87 \mathrm{E}+04$ & $1.38 \mathrm{E}+05$ & $6.86 \mathrm{E}+04$ & $6.40 \mathrm{E}+04$ & 0.41 & 0.31 & 0.33 & 0.36 \\
\hline 1 & 15 & 750 & 0.00002 & 0.001 & 0.25 & 0.002 & $6.70 \mathrm{E}+04$ & $1.80 \mathrm{E}+05$ & $8.93 E+04$ & $6.93 \mathrm{E}+04$ & 0.39 & 0.29 & 0.31 & 0.35 \\
\hline 1 & 15 & 750 & 0.00002 & 0.001 & 0.3 & 0.002 & $7.53 \mathrm{E}+04$ & $2.27 \mathrm{E}+05$ & $1.12 \mathrm{E}+05$ & $7.40 \mathrm{E}+04$ & 0.36 & 0.27 & 0.28 & 0.34 \\
\hline 1 & 15 & 750 & 0.00002 & 0.001 & 0.35 & 0.002 & $8.33 E+04$ & $2.78 \mathrm{E}+05$ & $1.37 \mathrm{E}+05$ & $7.84 \mathrm{E}+04$ & 0.35 & 0.25 & 0.27 & 0.34 \\
\hline 1 & 15 & 750 & 0.00002 & 0.001 & 0.4 & 0.002 & $9.12 \mathrm{E}+04$ & $3.34 \mathrm{E}+05$ & $1.64 \mathrm{E}+05$ & $8.25 E+04$ & 0.33 & 0.24 & 0.25 & 0.33 \\
\hline 1 & 15 & 750 & 0.00002 & 0.001 & 0.45 & 0.002 & $9.86 \mathrm{E}+04$ & $3.94 \mathrm{E}+05$ & $1.94 \mathrm{E}+05$ & $8.63 E+04$ & 0.32 & 0.22 & 0.24 & 0.33 \\
\hline 1 & 15 & 750 & 0.00002 & 0.001 & 0.5 & 0.002 & $1.06 \mathrm{E}+05$ & $4.58 \mathrm{E}+05$ & $2.25 \mathrm{E}+05$ & $8.99 \mathrm{E}+04$ & 0.31 & 0.21 & 0.23 & 0.32 \\
\hline 0.5 & 15 & 750 & 0.00002 & 0.001 & 0.02 & 0.002 & $8.25 E+04$ & $7.88 \mathrm{E}+04$ & $4.45 E+04$ & $1.25 \mathrm{E}+05$ & 0.69 & 0.56 & 0.61 & 0.48 \\
\hline 0.75 & 15 & 750 & 0.00002 & 0.001 & 0.02 & 0.002 & $3.61 \mathrm{E}+04$ & $3.66 \mathrm{E}+04$ & $2.05 \mathrm{E}+04$ & $5.77 \mathrm{E}+04$ & 0.69 & 0.55 & 0.59 & 0.47 \\
\hline 1 & 15 & 750 & 0.00002 & 0.001 & 0.02 & 0.002 & $1.99 \mathrm{E}+04$ & $2.17 \mathrm{E}+04$ & $1.21 \mathrm{E}+04$ & $3.34 \mathrm{E}+04$ & 0.70 & 0.54 & 0.56 & 0.47 \\
\hline 1.25 & 15 & 750 & 0.00002 & 0.001 & 0.02 & 0.002 & $1.26 \mathrm{E}+04$ & $1.47 \mathrm{E}+04$ & $8.23 E+03$ & $2.19 \mathrm{E}+04$ & 0.70 & 0.53 & 0.53 & 0.47 \\
\hline 1.5 & 15 & 750 & 0.00002 & 0.001 & 0.02 & 0.002 & $8.70 \mathrm{E}+03$ & $1.09 \mathrm{E}+04$ & $6.21 \mathrm{E}+03$ & $1.55 \mathrm{E}+04$ & 0.70 & 0.51 & 0.50 & 0.46 \\
\hline 1.75 & 15 & 750 & 0.00002 & 0.001 & 0.02 & 0.002 & $5.98 \mathrm{E}+03$ & $8.60 \mathrm{E}+03$ & $5.04 \mathrm{E}+03$ & $1.16 \mathrm{E}+04$ & 0.69 & 0.50 & 0.47 & 0.46 \\
\hline 2 & 15 & 750 & 0.00002 & 0.001 & 0.02 & 0.002 & $4.35 \mathrm{E}+03$ & $7.11 \mathrm{E}+03$ & $4.29 \mathrm{E}+03$ & $9.02 \mathrm{E}+03$ & 0.68 & 0.48 & 0.44 & 0.46 \\
\hline 1 & 15 & 750 & 0.00002 & 0.0001 & 0.02 & 0.002 & $4.12 \mathrm{E}+03$ & $8.74 E+03$ & $4.59 \mathrm{E}+03$ & $1.21 \mathrm{E}+04$ & 0.44 & 0.37 & 0.33 & 0.33 \\
\hline 1 & 15 & 750 & 0.00002 & 0.0003 & 0.02 & 0.002 & $8.32 \mathrm{E}+03$ & $1.27 E+04$ & $6.73 E+03$ & $1.96 \mathrm{E}+04$ & 0.56 & 0.44 & 0.43 & 0.39 \\
\hline 1 & 15 & 750 & 0.00002 & 0.0005 & 0.02 & 0.002 & $1.24 \mathrm{E}+04$ & $1.57 \mathrm{E}+04$ & $8.43 E+03$ & $2.45 \mathrm{E}+04$ & 0.63 & 0.48 & 0.49 & 0.42 \\
\hline 1 & 15 & 750 & 0.00002 & 0.0007 & 0.02 & 0.002 & $1.58 \mathrm{E}+04$ & $1.82 \mathrm{E}+04$ & $9.95 \mathrm{E}+03$ & $2.85 \mathrm{E}+04$ & 0.66 & 0.51 & 0.52 & 0.45 \\
\hline 1 & 15 & 750 & 0.00002 & 0.001 & 0.02 & 0.002 & $2.05 E+04$ & $2.17 \mathrm{E}+04$ & $1.21 \mathrm{E}+04$ & $3.34 \mathrm{E}+04$ & 0.70 & 0.54 & 0.56 & 0.47 \\
\hline 1 & 15 & 750 & 0.00002 & 0.002 & 0.02 & 0.002 & $3.40 \mathrm{E}+04$ & $3.13 E+04$ & $1.83 \mathrm{E}+04$ & $4.56 \mathrm{E}+04$ & 0.76 & 0.59 & 0.63 & 0.52 \\
\hline 1 & 15 & 750 & 0.00002 & 0.003 & 0.02 & 0.002 & $4.71 \mathrm{E}+04$ & $3.96 \mathrm{E}+04$ & $2.39 \mathrm{E}+04$ & $5.48 \mathrm{E}+04$ & 0.79 & 0.62 & 0.67 & 0.55 \\
\hline 1 & 15 & 750 & 0.00002 & 0.004 & 0.02 & 0.002 & $6.00 \mathrm{E}+04$ & $4.71 \mathrm{E}+04$ & $2.91 \mathrm{E}+04$ & $6.24 \mathrm{E}+04$ & 0.82 & 0.65 & 0.70 & 0.57 \\
\hline 1 & 15 & 750 & 0.00002 & 0.005 & 0.02 & 0.002 & $7.11 \mathrm{E}+04$ & $5.42 \mathrm{E}+04$ & $3.42 \mathrm{E}+04$ & $6.90 \mathrm{E}+04$ & 0.83 & 0.66 & 0.72 & 0.58 \\
\hline
\end{tabular}

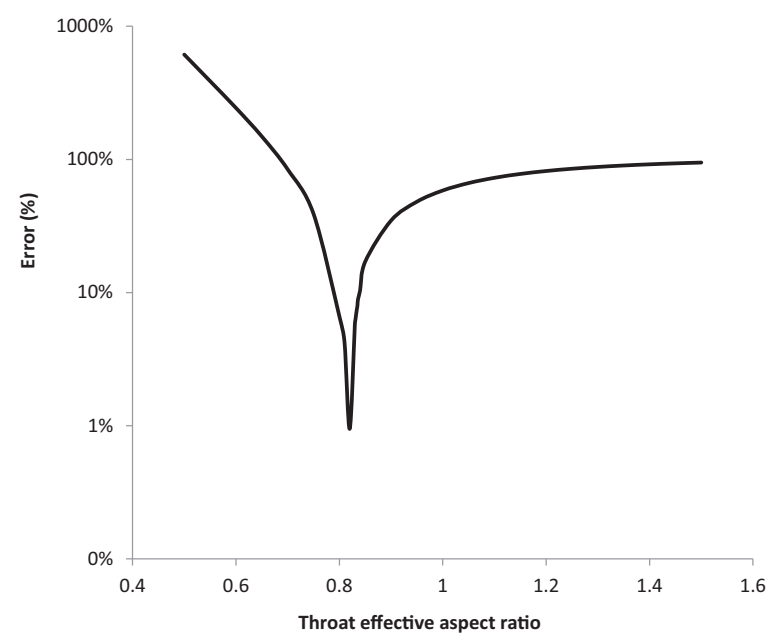

Fig. 4. Relative difference (error) between PNM pressure drop and the one calculated by Attou et al. model as a function of throat aspect ratio used in the PNM.

agreement with Attou al. model trends though its evolution keeps closer to Larachi et al. correlation and Holub et al. model. The behavior at the largest tested gas superficial velocity is closer to the Larachi et al. correlation. This presents the lowest mean deviation (17\%) over the whole range of $V_{s g}$. As only viscous contributions are accounted for in the PNM model, an underestimation of pressure drop is not surprising at high gas velocity, i.e., when the gas phase inertial friction contribution is no longer negligible. Furthermore, the gas phase Reynolds number is equal to 75 at $V_{\mathrm{sg}}=0.1 \mathrm{~m} / \mathrm{s}$ which may explain the strong discrepancy with regard to Attou et al. model. The crossover at high gas velocities between PNM and Holub et al. model simulations is noteworthy. The variety of trends predicted in gas inertia-dominated flows by the three benchmark estimation methods (concave and convex shapes) may highlight the necessity to incorporate in future PNM model versions the effect of inertial forces especially in the gas phase. Such an extension has been carried out successfully for the one-phase flow case [28].

\subsection{Effect of diameter}

Particle diameters, $d$, from 0.5 to $2 \mathrm{~mm}$ are used to compare model predictions. For all of them, the effect of $d$ on the pressure drop is very important obeying roughly a $\sim d^{-4}$ power law. An excellent agreement between PNM and Attou et al. model is observed over the whole range at $d=1 \mathrm{~mm}$ signifying much likely that weak gas inertial effects prevail in these conditions while representation of the Darcy-regime adjusted throat aspect ratio, described above, is realistic. Maximum deviation is observed for $d=2 \mathrm{~mm}$ where the PNM result is closer to that of Holub et al. 


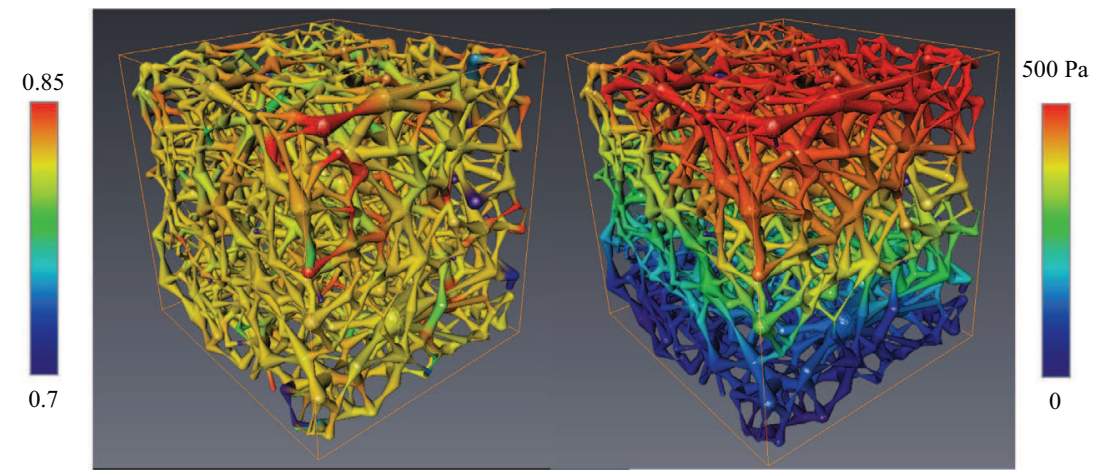

Fig. 5. Example of liquid throat saturation and pressure result in the network (case of $1 \mathrm{~mm}$ particles, $V_{\mathrm{sl}}=2 \mathrm{~mm} / \mathrm{s}, V_{\mathrm{sg}}=2 \mathrm{~cm} / \mathrm{s}$ ).

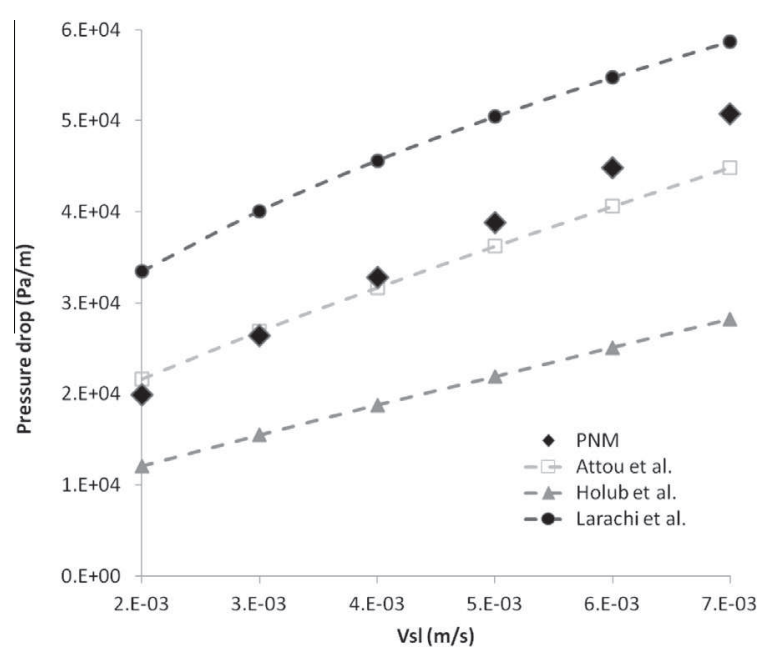

Fig. 6. Comparison of pressured drops calculated by PNM, Attou et al., Holub et al. and Larachi et al. estimation methods versus superficial liquid velocity.

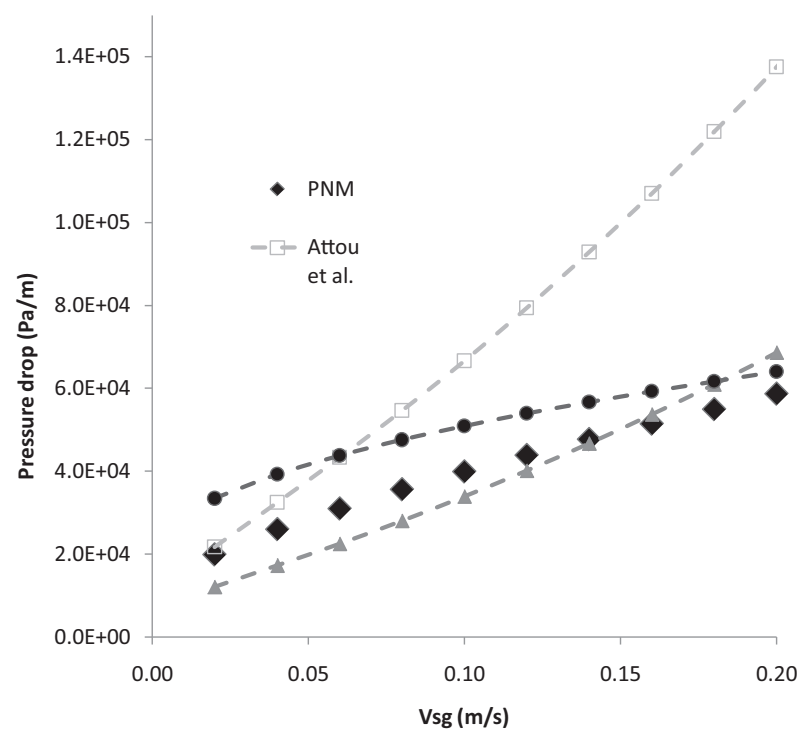

Fig. 7. Comparison of pressure drops calculated by PNM, Attou et al., Holub et al. and Larachi et al. estimation methods versus superficial gas velocity.

model. The mean deviation between PNM and Attou et al. model is ca. $15 \%$. Fig. 8 portrays the dependence with respect to the particle diameter of the various models.

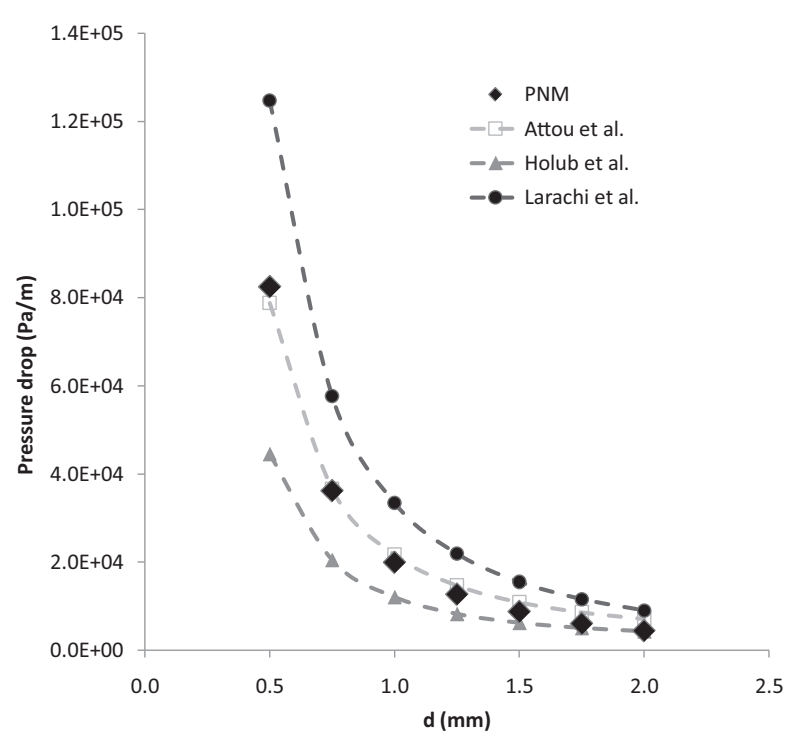

Fig. 8. Comparison of pressure drops calculated by PNM, Attou et al., Holub et al. and Larachi et al. estimation methods versus particle diameter.

\subsection{Effect of the fluid viscosity}

The effect of liquid viscosity is studied in the range of $\mu_{L}\left[10^{-4}\right.$ to $5.10^{-3}$ ] to mimic petroleum hydrorefining liquid cuts. The simulation results are reported in Fig. 9. The minimal mean deviation between PNM and existing models is observed with the Attou et al. model (24\%) with a crossover around $1 \mathrm{cp}$ liquid viscosity. PNM pressure drop predictions lie below those from Attou et al. before the crossover point and vice versa. This trend may be attributed to the neglect of the inertial effects from both gas and liquid phases, despite the Reynolds number of the liquid phase kept always very low $\left(\operatorname{Re}_{\mathrm{L}}<15\right)$. However, the PNM overestimating trend against Attou et al. model for liquid viscosities in excess of $1 \mathrm{cp}$ (Fig. 9) might point to the approximate character of our hypothesis of a viscosity-indifferent correction of the throat effective aspect ratio as calibrated with $1 \mathrm{cp}$ liquid at $V_{\mathrm{sl}}=0002 \mathrm{~m} / \mathrm{s}$ (Fig. 4).

\subsection{Liquid saturation}

In its formulation in the present study, the pore network model involves only the physics in the throat between pores in the form of fully developed segregated gas-liquid flows. As a consequence, the phase saturations calculated from the model are not representative of the various fluid-bearing capacities in the fixed bed except 


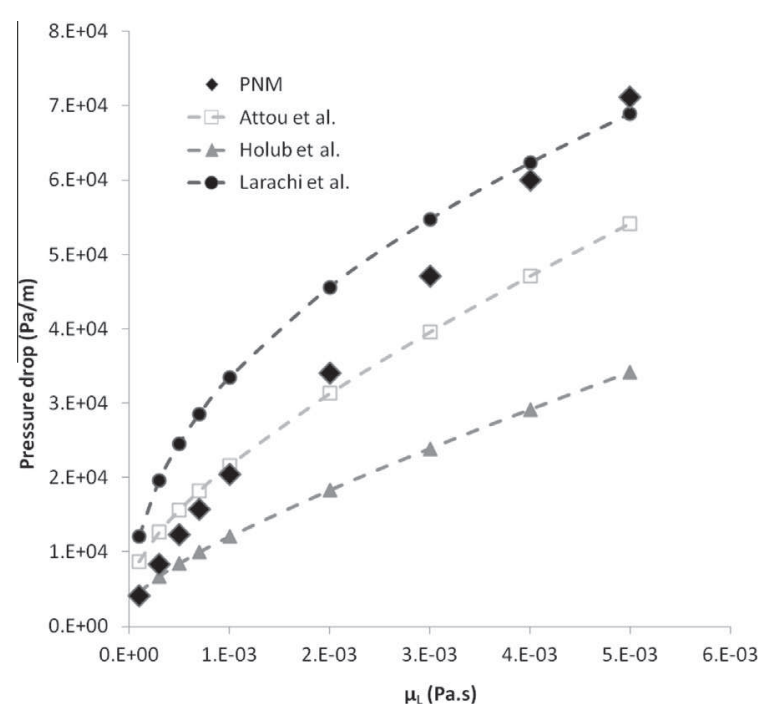

Fig. 9. Comparison of pressure drops calculated by PNM, Attou et al., Holub et al. and Larachi et al. methods versus liquid dynamic viscosity.

that of the throats. For instance, pore saturations were not explicitly handled in the current PNM formulation and therefore reliance on the model predicted liquid saturations would simply be premature. Nevertheless, for qualitative comparative purposes, PNM liquid saturations and total liquid saturations calculated by the three benchmark models are compared in Fig. 10 as a function of $V_{\mathrm{sl}}$. The liquid saturation calculated by the PNM follows the same trend as the other models though by showcasing systematically higher values. A physical explanation could be that the pores contain much more gas than the channels contrary to the predictions suggested by Eq. (14). An empirical correction of the PNM liquid saturation was thus proposed to estimate a posteriori the total liquid saturation in the bed. The average liquid saturation calculated by PNM $\varepsilon_{L}$ is simply multiplied by a correction function of the average particle diameter $d$. Parameters of the correction function are chosen to fit the corrected liquid saturation $\varepsilon_{C}$ with Attou model. $\varepsilon_{C}$ is only a post-treated value, it is not used to solve PNM.

$\varepsilon_{C}=0.36 \varepsilon_{L} d^{-0.11}$

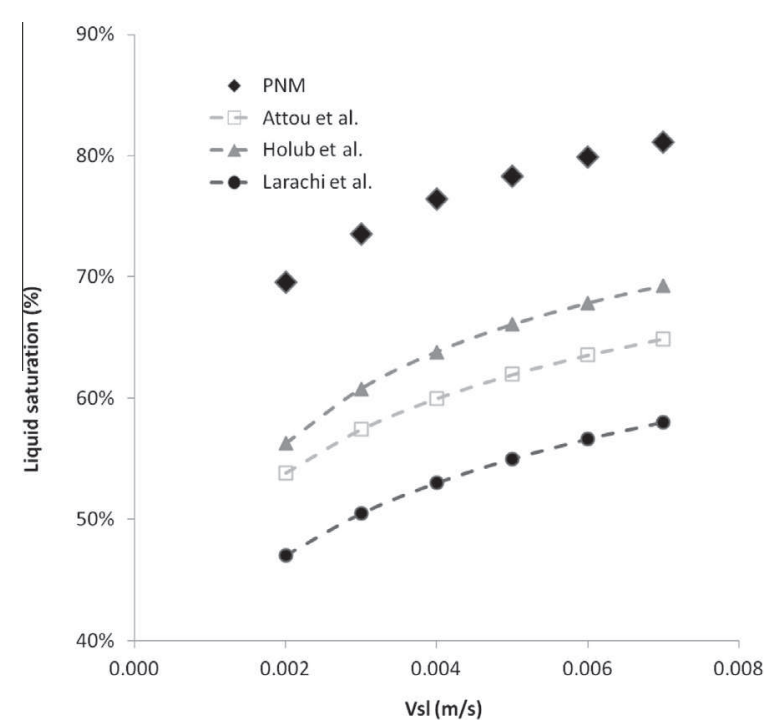

Fig. 10. Comparison of liquid saturation calculated by PNM, Attou et al., Holub et al. and Larachi et al. estimation methods versus superficial liquid velocity.

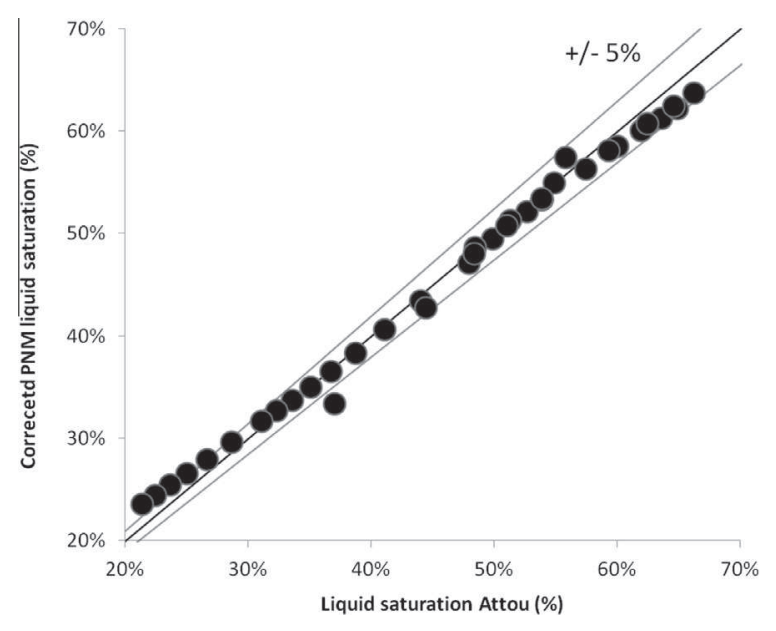

Fig. 11. Parity diagram of corrected liquid saturation calculated with PNM versus Attou et al. model.

More physical models based on the liquid thickness and the shape of throats and pores may be developed, but, in spite of its relative simplicity, Eq. (16) provides results very close to Attou et al. model for any of the tested conditions of Table 1 as illustrated in Fig. 11. The mean relative error is $2.5 \%$. The good agreement between the Attou et al. model and the PNM-modified liquid saturation shows that the friction at the throat between pores has a major importance on the total phase volume fraction. The incorporation of the saturation correction in the model is certainly possible and would be necessary in many instances, in particular when coupling the two-phase model with the transport of any chemical species, since accumulation terms in the balance equations play an important role in that case.

\section{Conclusion}

A pore network model has been developed to predict trickling gas-liquid flows in fixed bed reactors. The network geometry has been obtained from previous X-ray micro-tomography experiments [28] performed on a sample of spherical particles. The physics employed to describe the two-phase flow is deduced from the solution of fully developed (gas) core/(liquid) annulus viscous flows in cylindrical tubes. This so-called "two-fluid Poiseuille velocity profile" possesses an explicit analytical solution that can be easily applied to each individual channel of the network. The link between pores and channels is realized by constraining the pore saturations to be equal to the interconnected downstream channels. This simplified relation is usable in the case of homogeneous flows but may be insufficient to predict non-homogeneous flows such as liquid jets at the top of the domain, because the jet dispersion may probably be overestimated with the involved model.

When compared to some existing pressure drop models of interest, good agreement is found in a large range of fluid flow rates and physical properties. The results are globally close to Attou et al. model, except at high gas velocity. This limitation of the model is directly linked to the hypothesized viscous Poiseuille flows assumed in all the channels.

Nevertheless, the presented approach does not aim to replace existing 1D models which are still the most relevant tools for engineers in terms of tradeoff between engineering accuracy and computational complexity. The PNM approach requires the knowledge of complex fixed bed geometries and the network extraction is not easy. However, this extra-cost is rewarded by more insights into the physics at play at the local level. In that sense, foreseeing 
construction of macroscopic closure forms from ensemble averaging pore network information is promising. But as the PNM physics behaves well in homogeneous flows, perspectives of this work are to adapt the model to non-homogeneous flows in order to characterize dispersion phenomena. This would take advantage of the multidimensional aspect of PNM.

Finally, the liquid saturation in a throat is actually deduced directly from its value in the upstream pore, regardless of the orientation of the throats; this point may need improvements to predict well heterogeneous flows as liquid jet spreading.

\section{References}

[1] P.A. Ramachandran, R.V. Chaudhari, Topics in chemical engineering, ThreePhase Catalytic Reactors, vol. 2, Gordon and Breach Science Publishers, 1983.

[2] C. Julcour-Lebigue, F. Augier, H. Maffre, A.M. Wilhelm, H. Delmas, Measurements and modeling of wetting efficiency in trickle-bed reactors: liquid viscosity and bed packing effects, Ind. Eng. Chem. Res. 48 (2009) 68116819.

[3] F. Augier, A. Koudil, A. Royon-Lebeaud, L. Muszynski, Q. Yanouri, Numerical approach to predict wetting and catalyst efficiencies inside trickle bed reactors, Chem. Eng. Sci. 65 (2010) 255-260.

[4] R.N. Maiti, K.D.P. Nigam, Gas-liquid distributors for trickle-bed reactors: a review, Ind. Eng. Chem. Res. 46 (2007) 6164-6182.

[5] F. Bazer-Bachi, Y. Haroun, F. Augier, C. Boyer, Experimental evaluation of distributor technologies for trickle-bed reactors, Ind. Eng. Chem. Res. 52 (2013) 11189-11197.

[6] C. Marcandelli, A.S. Lamine, J.R. Bernard, G. Wild, Liquid distribution in tricklebed reactor, Oil Gas Sci. Technol. - Rev. IFP 55 (2000) 407-415.

[7] K. Lappalainen, M. Manninen, V. Alopeus, CFD modeling of radial spreading of flow in trickle-bed reactors due to mechanical and capillary dispersion, Chem. Eng. Sci. 64 (2009) 207-218.

[8] Y. Wang, J. Chen, F. Larachi, Modelling and simulation of trickle-bed reactors using computational fluid dynamics: a state-of-the-art review, Can. J. Chem. Eng. 91 (2013) 136-180.

[9] R.J.G. Lopes, R.M. Quinta-Ferreira, Assessment of CFD-VOF method for tricklebed reactor modelling in the catalytic wet oxidation of phenolic wastewaters, Ind. Eng. Chem. Res. 49 (6) (2010) 2638-2648.

[10] Y. Haroun, L. Raynal, P. Alix, Prediction of effective area and liquid hold-up in structured packings by CFD, Chem. Eng. Res. Des. (2014), http://dx.doi.org/ 10.1016/j.cherd.2013.12.029.

[11] F. Larachi, A. Laurent, N. Midoux, G. Wild, Experimental study of a trickle bed reactors operating at high pressure: two phase pressure drop and liquid saturation, Chem. Eng. Sci. 46 (1991) 1233.

[12] M.H. Al-Dahhan, M.R. Khadilkar, Y. Wu, M.P. Dudukovic, Prediction of pressure drop and liquid holdup in high-pressure trickle-bed reactors, Ind. Eng. Chem. Res. 37 (1998) 793-798

[13] A. Attou, C. Boyer, G. Ferschneider, Modelling of the hydrodynamics of the cocurrent gas-liquid trickle flow through a trickle-bed reactor, Chem. Eng. Sci. 54 (1999) 785-802.

[14] I. Iliuta, F. Larachi, M.H. Al-Dahhan, A multiple-zone model for partially wetted trickle flow hydrodynamics, Chem. Eng. Res. Des. (Trans IchemE) 78 (2000) 982-989.

[15] R.A. Holub, M.P. Duduković, P.A. Ramachandran, A phenomenological model for pressure drop, liquid holdup, and flow regime transition in gas-liquid trickle flow, Chem. Eng. Sci. 47 (1992) 2343-2348.

[16] F. Larachi, Z. Bensetiti, B.P.A. Grandjean, G. Wild, Two-phase frictional pressure drop in flooded-bed reactors: a state-of-the-art correlation, Chem. Eng. Technol. 21 (1998) 887-893.

[17] A.J. Sederman, L.F. Gladden, Magnetic resonance imaging as a quantitative probe of gas-liquid distribution and wetting efficiency in trickle-bed reactors, Chem. Eng. Sci. 56 (2001) 2615-2628.

[18] M. Schubert, G. Hessel, C. Zippe, R. Lange, U. Hampel, Liquid flow texture analysis in trickle bed reactors using high-resolution gamma ray tomography, Chem. Eng. J. 140 (2008) 332-340.

[19] W. van der Merwe, W. Nicol, F. de Beer, Three-dimensional analysis of trickle flow hydrodynamics: computed tomography image acquisition and processing, Chem. Eng. Sci. 62 (2007) 7233-7244.
[20] P. Horgue, F. Augier, P. Duru, M. Quintard, M. Prat, Arrays of cylinders as an experimental and numerical laboratory for the study of trickling and pulsing flows, Chem. Eng. Sci. 102 (2013) 335-345.

[21] M.J. Blunt, M.D. Jackson, M. Piri, P.H. Valvatne, Detailed physics, predictive capabilities and macroscopic consequences for pore-network models of multiphase flows, Adv. Water Resour. 25 (2002) 1069-1089.

[22] I. Fatt, The network model of porous media I. Capillary pressure characteristics, Pet. Trans. AIME 207 (1956) 144-159.

[23] S. Bryant, M. Blunt, Prediction of relative permeability in simple porous media, Phys. Rev. A 46 (1992) 2004-2011.

[24] M.A. Ioannidis, I. Chatzis, Network modeling of pore structure and transport properties of porous media, Chem. Eng. Sci. 48 (1993) 951-972.

[25] T.W. Patzek, Verification of a complete pore network simulator of drainage and imbibition, SPE J. 6 (2001) 144-156.

[26] K.E. Thompson, H.S. Fogler, Modeling flow in disordered packed beds from pore-scale fluid mechanics, AIChE J. 43 (1997) 1377-1389.

[27] C. Jia, K. Shing, Y.C. Yortsos, Advective mass transfer from stationary sources in porous media, Water Resour. Res. 35 (1999) 3239-3251.

[28] F. Larachi, R. Hannaoui, P. Horgue, F. Augier, Y. Haroun, S. Youssef, E. Rosenberg, M. Prat, M. Quintard, X-ray micro-tomography and pore network modeling of single-phase fixed-bed reactors, Chem. Eng. J. 240 (2014) 290306.

[29] M. Blunt, M.D. Jackson, M. Piri, P.H. Valvatne, Detailed physics, predictive capabilities and macroscopic consequences for pore-network models of multiphase flow, Adv. Water Resour. 25 (2002) 1069-1089.

[30] P.H. Valvatne, M.J. Blunt, Predictive pore-scale modelling of two-phase flow in mixed wet media, Water Resour. Res. 40 (2004) W07406.

[31] V. Joekar-Niasar, S.M. Hassanizadeh, A. Leijnse, Insights into the relationships among capillary pressure, saturation, interfacial area and relative permeability using pore-network modelling, Transp. Porous Media 74 (2) (2008) 201-219.

[32] V. Joekar-Niasar, S.M. Hassanizadeh, L.J. Pyrak-Nolte, C. Berentsen, Simulating drainage and imbibition experiments in a high-porosity micromodel using an unstructured pore network model, Water Resour. Res. 45 (2009) W02430.

[33] J. Koplik, T.J. Lasseter, 1 Two-phase flow in random network models of porous media, Soc. Petrol. Eng. J. 25 (1985) 89-110.

[34] E.K. Aker, J. Maloy, A. Hansen, G.G. Batrouni, A two-dimensional network simulator for two-phase flow in porous media, Transp. Porous Media 32 (1998) 163-186.

[35] H.K. Dahle, M.A. Celia, A dynamic network model for two-phase immiscible flow, Comput. Geosci. 3 (1999) 1-22.

[36] M.S. Al-Gharbi, M.J. Blunt, Dynamic network modelling of two-phase drainage in porous media, Phys. Rev. E 71 (2005) 016308.

[37] M. Prat, Recent advances in pore-scale models for drying of porous media, Chem. Eng. J. 86 (2002) 153-164.

[38] V. Joekar-Niasar, S.M. Hassanizadeh, H.K. Dahle, Non-equilibrium effects in capillarity and interfacial area in two-phase flow: dynamic pore-network modelling, J. Fluid Mech. 655 (2010) 38-71.

[39] H.N. Man, X.D. Jing, Network modeling of wettability and pore geometry effects on electrical resistivity and capillary pressure, J. Petrol. Sci. Eng. 24 (1999) 255-267.

[40] M. Blunt, P. King, Relative permeabilities from two- and three-dimensional pore-scale network modelling, Transp. Porous Media 6 (1991) 407-433.

[41] S. Youssef, E. Rosenberg, N. Gland, S. Bekri, O. Vizika, Quantitative 3D Characterisation of the Pore Space of Real Rocks: Improved l-CT Resolution and Pore Extraction Methodology, Int. Sym. Soc. Core Analysts, Calgary, Sept 10-12 (2007) paper SCA2007-17.

[42] S. Youssef, M. Han, D. Bauer, E. Rosenberg, S. Bekri, M. Fleury, O. Vizika. High Resolution l-CT Combined to Numerical Models to Assess Electrical Properties of Bimodal Carbonates, Int. Sym. Soc. Core Analysts, Abu-Dhabi, UAE, 29 October-2 November, 2008, paper SCA2008-37.

[43] M. Singh, K.K. Mohanty, Dynamic modelling of drainage through three dimensional porous materials, Chem. Eng. Sci. 58 (2003) 1-18.

[44] Y. Saad, M.H. Schultz, Gmres: a generalized minimal residual algorithm for solving nonsymmetric linear systems, SIAM J. Sci. Stat. Comput. 7 (1986) 856869.

[45] K. Mogensen, E.H. Stenby, A dynamic two-phase pore-scale model for imbibition, Transp. Porous Media 32 (1998) 299-327.

[46] G.F. Pinder, W.G. Gray, Essentials of Multiphase Flow and Transport in Porous Media, John Wiley \& Sons Inc, New Jersey, USA, 2008.

[47] V. Joekar-Niasar, M. Prodanovic, D. Wildenschild, S.M. Hassanizadeh, Network model investigation of interfacial area, capillary pressure and saturation relationships in granular porous media, Water Resour. Res. 46 (W06526) (2010) 1-18. 